\title{
PRIMER ANÁLISIS DEL PROBLEMA DE LA FUNDAMENTACIÓN ÚLTIMA FILOSÓFICA EN K. O. $\mathrm{APEL}^{1}$
}

\author{
Ricardo Navia \\ Universidad de la República-Uruguay \\ naviamar adinet.com.uy
}

\begin{abstract}
Resumen: Dada la complejidad y vastas implicaciones del tema abordado por Apel en su trabajo (aún no traducido al portugués): El problema de la fundamentación última filosófica a la luz de una pragmática trascendental del lenguaje (1976) - un verdadero tour de force desde su propio título -, el presente artículo, como "primer análisis" del mismo, solo se propone ubicar y analizar las principales etapas y estrategias argumentativas usadas por el autor, así como indicar sus más importantes apoyos teóricos.
\end{abstract}

Palabras claves: Trilema de Münchhausen, reflexión pragmático-trascendental, fundamentación filosófica no-metafísica.

LA NEGACIÓn DEL FundAMENTACIONISMO POR HANS AlberT

Apel comienza su ensayo reseñando la más reciente versión de la tesis de la imposibilidad de una fundamentación última filosófica desarrollada por los filósofos del llamado "racionalismo crítico": William N. Bartley y Hans Albert, con inspiración en la "Lógica de la investigación científica" de K. Popper, así como su propuesta de sustituir el programa de fundamentación última por el programa de una crítica racional ilimitada. Reconoce en H. Albert a quien ha defendido especialmente este programa de alternativa en su Tratado de la razón crítica (1976).

Albert desarrolla tal operación crítica mediante la deducción del llamado “Trilema de Münchhausen”. Según Albert, todo intento de fundamentación filosófica última conduce ineludiblemente: o bien (1) a un regreso al infinito, irrealizable por definición; o bien (2) a un círculo lógico, en el cual la fundamentación recurre en 
algún momento a una aserción que ya se había visto que carecía de fundamentación; o bien (3) a una interrupción arbitraria del razonamiento que implicaría una abdicación total. ${ }^{2}$

Siendo las tres únicas opciones, inaceptables; nos encontramos ante un verdadero trilema, que demostraría la imposibilidad del proyecto de fundamentación. Albert concluye que no sólo tal objetivo es inalcanzable, sino que además es incompatible con la búsqueda de la verdad; desde su perspectiva, la "voluntad de certeza" es contraria a la "voluntad de conocimiento".

A partir de este planteamiento Albert propone, siguiendo a Popper, tomar una decisión - imposible de fundamentar enteramente de forma racional - a favor del "principio del falibilismo", y, concomitantemente, a favor de un método que no sustraiga ningún conocimiento a la crítica y que, en cambio, proporcione a la realidad oportunidad de "hacerse valer decisivamente" (Albert, 1976, p. 33).

Esta posición tiene no sólo importantes consecuencias para el examen de teorías científicas sino, más allá, una notoria significación ética, social y política. Proponen someter todo sistema moral a un permanente examen crítico, convenientemente a la luz de alternativas.

Sobre LAS CONDICIONES DE POSIBILIDAd DE UNA CRÍTICA INTERSUBJETIVAMENTE VÁLIDA Y LA NECESIDAD DE CONSIDERAR LA DIMENSIÓN PRAGMÁTICA

En su trabajo Apel se propone someter al "racionalismo crítico" a un examen metacrítico (no cuestiona, como era de esperar, su principio de criticidad), preguntando por las "condiciones de posibilidad de una crítica intersubjetivamente válida”, es decir, por las condiciones de posibilidad del examen crítico tanto de conocimiento científico como de normas morales.

Más exactamente - dice Apel - debe investigarse si [...] el principio de fundamentación o justificación puede ser 
sustituido por el principio de la crítica, o si [...] el principio de fundamentación más bien no es presupuesto por el principio de la crítica válida intersubjetivamente. (APEL, 1975, p. 143)

Apel empieza su argumentación recordando que desde el pensamiento clásico antiguo, el problema de la fundamentación última filosófica se planteó en íntima vinculación con el conocimiento de la imposibilidad de una fundamentación apodíctico-deductiva de los primeros (o últimos) principios (axiomas del pensamiento lógico-matemático). Esto es especialmente claro en Aristóteles (Metafísica 4, 1006 a 6-18).

Pero la concepción de Aristóteles de los axiomas como principios inmediatamente evidentes, llevada a la justificación filosófica por Descartes, da lugar a la idea de que la fundamentación filosófica total debe apelar en última instancia a la evidencia de conciencia (Principia I, 11.52); con lo cual, el problema no puede concebirse ya como un problema puramente lógico-formal. Es decir, que "a diferencia de la problemática lógico-matemática [...] de la fundamentación última, el principio moderno de razón suficiente, en cuanto exige el recurso a la evidencia [...], es de antemano un problema epistemológico" (APEL, 1975, p. 145); esto es, un principio que involucra la dimensión pragmática en tanto se trata de la evidencia para un sujeto de conocimiento.

Pero, a su vez, Apel no concibe las condiciones pragmáticas de posibilidad del conocimiento científico como condiciones contextuales sin incidencia para la validez del conocimiento, sino al menos en parte en el sentido de Kant- como condiciones de posibilidad del conocimiento (y de la crítica) científico y filosófico intersubjetivamente válidos.

Como ya la señalara Charles S. Peirce, es una implicación lógica de la tridimensionalidad de la relación sígnica que las funciones interno-sintácticas y semántico-referenciales presuponen el presupuesto de un uso e interpretación (pragmática) de esos signos 
a cargo de alguna comunidad semiótica determinada. Es por ello que Apel, siguiendo a Peirce, ${ }^{3}$ propone una "pragmática trascendental del lenguaje" como una "reflexión sobre las condiciones de posibilidad del conocimiento lingüísticamente formulado, y en cuanto tal, intersubjetivamente válido” (APEL, 1975, p. 147).

Necesidad de una PRAgmática - trascendental del lenguaje COMO NUEVA FUENTE DE EVIDENCIA

De esta convergencia reconstructiva del pragmatismo de Peirce con el trascendentalismo de Kant, se deriva una "transformación semiótica de la filosofía trascendental”, que se ocupará de la reflexión sobre las condiciones de posibilidad y validez de las convenciones; una pragmática trascendental del lenguaje como metadisciplina ya no formalizable. En nuestro contexto polémico, Apel intentará aplicarla al examen de las "condiciones de posibilidad de una crítica intersubjetivamente válida”. En especial, el Trilema de Münchhausen de la fundamentación suficiente solo es aplicable en relación a sistemas de proposiciones (axiomáticamente ordenados) como sistemas exclusivamente sintáctico-semánticos, es decir, haciendo abstracción de la dimensión pragmática del lenguaje argumentativo. Sólo entonces, el recurso a la evidencia puede catalogarse como interrupción del proceso de justificación.

En cambio, desde un enfoque pragmático-trascendental, el procedimiento de derivación de proposiciones a partir de proposiciones es concebido desde el principio, sólo como una etapa intermedia de la fundamentación argumentativa de enunciados, basada -esta última en definitiva-, en evidencia a priori intersubjetiva. Esto no significa, para Apel, que tales evidencias cognoscitivas puedan ser tomadas como base indubitable para asertos científicos independientemente del lenguaje.

Basándose en Searle, Apel sostiene, que a partir de los "actos proposicionales" identificadores de referencia y sentido, las 
evidencias cognoscitivas están desde siempre entretejidas con el uso del lenguaje ("actos de habla"), y con la praxis de los sujetos, en “juegos de lenguaje”, como lo mostró el último Wittgenstein. Sin este entretejido, a priori, pragmático-trascendental- no sería posible ni el aprendizaje del lenguaje, ni el de conductas, ni la comunicación misma. Y son: "evidencias experienciales paradigmáticas”, luego necesariamente compartidas, las que ofician de gozne de tal entretejido.

A partir de esto, Apel entiende que la fundamentación de la validez del conocimiento no puede darse ni por la deducción lógica de proposiciones ni apelando a evidencia intuitiva de conciencia (supuestamente independiente del lenguaje), sino que:

Más bien la fundamentación, en cuanto lo es de la validez del conocimiento, tiene que apoyarse siempre al mismo tiempo sobre las posibles evidencias de conciencia de los sujetos cognoscentes individuales... y sobre las reglas a priori intersubjetivas de un discurso de argumentación, en cuyo contexto las evidencias de conocimiento, como testimonios subjetivos de validez objetiva, tienen que alcanzar una validez intersubjetiva. (APEL, 1975, p. 152)

Sin embargo, es fácil prever que Albert podría aún objetar que una tal fundamentación en base a evidencia, sigue ciñéndose al paradigma del racionalimo clásico de justificación última mediante una certeza indubitable, lo cual es claramente contrario con el principio de falibilismo. Apel se ve necesitado entonces de argumentar contra el propio principio del falibilismo, o mejor aún, contra su aplicación ilimitada.

¿Principio de falibilismo O POSIBILIDAd dE Fundamentación?: LAS CONDICIONES DE UNA "DUDA RAZONABLE”

Apel comienza recordando que el propio mentor del principio de falibilismo, Ch. S. Peirce, objetó en Descartes que quien dudara 
de todo caería en una duda vacía de contenido. Una "duda razonable", esto es, una duda significativa, supone que no se dude de todo, sino que se parta de ciertas convicciones que se aceptan como criterio para la duda y para la aceptación o rechazo de eventuales respuestas. Posiciones análogas aparecen en el Wittgenstein posterior. ${ }^{4}$

Radicalizando esta observación: todo juego lingüístico que funcione presupone que los interlocutores comparten determinadas creencias. Serán esas convicciones compartidas e indubitables las que operan como paradigmas del uso lingüistico dotado de sentido. Tales presupuestos son condiciones de posibilidad de todo juicio y de toda argumentación, incluso condiciones ineludibles para la duda y la crítica con sentido. También aquí Apel puede apoyarse en Wittgenstein. ${ }^{5}$

De aquí se deriva - en la argumentación de nuestro autor que la crítica no puede, cómo pretenden Albert y Bartley, ser última instancia de argumentación racional: ella supone siempre un marco pragmático-trascendental, un juego significativo de lenguaje, que incluye numerosas evidencias paradigmáticas que vinculan posibles requerimientos críticos con posibles justificaciones. Nuevamente aquí Wittgenstein acompaña la concepción "crítico-significativa": Leemos en el Aforismo 15 "Sobre la certeza":

Toda prueba, todo corroborar e invalidar una suposición sucede ya dentro de un sistema. Y este sistema, por cierto, no es un punto de arranque más o menos arbitrario o dudoso de nuestros argumentos, sino que pertenece a la esencia de lo que llamamos un argumento.

Observemos que, si tenemos en cuenta que las convenciones se forjan y rigen siempre en el interior de una comunidad, que ellas no son nunca un patrimonio de sujetos aislados; tenemos entonces que la existencia de una "comunidad de comunicación" es también una condición de posibilidad para la existencia de signos significativos, para la formulación de aserciones y para todo intento 
de fundamentación. Observamos asimismo que, a diferencia de Kant que fue favorable a una teoría de la verdad como correspondencia, Apel siguiendo a Peirce, se va a inclinar por una teoría pragmática de la verdad como consenso en el seno de una comunidad de comunicación. ${ }^{6}$

En síntesis, Apel descarta así, tanto la tesis del racionalismo pancrítico de la asimilación de la fundamentación por recurso a la evidencia con la apelación a un dogma (o con una interrupción arbitraria), como la idea de la prioridad del principio del falibilismo sobre la idea de la fundamentación. ${ }^{7}$

Esto no puede hacer olvidar que Peirce establece que, si bien en la ciencia no se puede duda de todo simultáneamente, sí se puede hacerlo individualmente de cada una de las creencias aceptadas; y ahí está su principio del falibilismo justificando una duda ahora “virtualmente universal”.¿Cómo se concilian el falibilismo virtualmente universal de Peirce con su pragmatismo de la certeza? Desde este punto, donde parece definitivamente arrinconado, Apel hará partir el trecho final de su argumentación.

\section{ESPECIFICIDAD DEL JUEGO LINGÜISTICO DE LA REFLEXIÓN FILOSÓFICA}

Según Apel la consistencia de falibilismo y certeza fundamentante, puede entenderse a partir de una distinción basada en el diferente grado reflexivo de: por una parte los juegos lingüísticos científicos y precientíficos, y, por otra, una reflexión pragmático-trascendental sobre la estructura de los propios juegos lingüísticos. Los primeros con una pretensión de validez individual o empírico-general, y los segundos con una pretensión de validez universal. Desde el punto de vista filosófico, se puede afirmar, con respecto a cualquier juego de lenguaje - incluído el filosófico -, que en su contexto la duda y la crítica sólo tienen sentido apelando a una reserva de evidencia paradigmática indubitable. Pero también en ese plano, se puede sostener la posibilidad de la duda virtualmente universal en relación a las evidencias paradigmáticas 
de todos los juegos del lenguaje - excepto del juego lingüístico filosófico de la duda. Sería una reconstrucción pragmático trascendental del cogito cartesiano.

Esta "reserva de falibilismo" expresa y fundamenta la posibilidad de una duda virtualmente universal; pero asimismo, el principio de falibilismo debe asentarse en una única certeza ya no cuestionable. Visto que las evidencias presupuestas en las argumentaciones de los juegos lingüísticos particulares pueden siempre ser revisadas, la crítica permanente mantiene entonces la última palabra en el grado de reflexión filosófica. Pero, razona Apel, si esto es así para el conocimiento científico, no demuestra empero la prioridad del principio falibilista en el terreno filosófico; sino más bien lo contrario, pues para que el principio rija en el campo científico (y para buena parte del campo filosófico) es necesario admitir que el juego filosófico de lenguaje (que lo sustenta) puede recurrir a evidencias ya no revisables.

DE LA REFLEXIÓN PRAGMÁtICO-TRASCENDENTAL SOBRE LA ARGUMENTACIÓNFILOSÓFICA A LA FUNDAMENTACIÓN ÚLTIMA CON BASE EN LOS PRESUPUESTOSDE TAL ARGUMENTACIÓN

Precisamente, en la última etapa del trabajo, Apel va a obtener el "material" de fundamentación última filosófica mediante una reflexión pragmático-trascendental sobre esas evidencias paradigmáticas del juego lingüístico-filosófico como condiciones de posibilidad de la validez intersubjetiva de la argumentación filosófica.

A diferencia de Kant, para nuestro autor es imposible saber con exactitud cuáles de los logros racionales pertenecen al núcleo filosófico-trascendental y cuáles al conjunto de los resultados revisables. Sabemos hoy, por ejemplo: que la demostrabilidad metalógica y metamatemática de la consistencia de sistemas lógicomatemáticos axiomatizados es necesariamente incompleta (GÖDEL, 1931); pero a su vez, ese mismo teorema nos proporciona el 
conocimiento reflexivo-trascendental sobre las condiciones de posibilidad (y los límites) de fundamentación en tales sistemas.

A propósito de esto Apel señala, en una interesantísima historización de la concepción trascendental:

[...] la exigencia kantiana de perfección definitiva de un "sistema de la razón pura" no puede aquí sostenerse; más bien se trata de un descubrimiento reflexivo progresivo de horizontes trascendentales, que se amplían en la medida en que también se amplía el conocimiento humano, al que debe interrogarse por sus condiciones de posibilidad. (APEL, 1975, p. 163)

Tales limitaciones no demuestran empero la imposibilidad de una fundamentación última. Para empezar, el principio del falibilismo no resiste la auto-aplicación: ya que si el principio mismo fuera falible, entonces tendríamos ya una evidencia. Lo cual muestra que, por lo menos, no puede aplicarse a la evidencia filosófica sobre la que él mismo se fundamenta.

Una vez así precisado el alcance (real) del principio de falibilismo, Apel intentará determinar, qué pertenece a esas condiciones de posiblidad no dubitables de una crítica y autocrítica filosófica intersubjetivamente válidas. Los primeros elementos Apel los va a tomar de una "confesión de parte" del propio W. Bartley (luego desarrollada por Hans Lenk, 1973, p. 105-107). En efecto, Bartley demostró que notoriamente la lógica "no puede pertenecer a aquella totalidad que debe ser sometida a prueba" puesto que "el ejercicio de una discusión crítica y la lógica están ligadas inseparablemente". 8

De este modo el remanente pragmático-trascendental del juego lingüístico filosófico se muestra como materia para una fundamentación última filosófica, en tanto sus evidencias paradigmáticas no pueden ser cuestionadas sin autocontradicción ni afirmadas deductivamente sin presuponerlas. Los defensores de la exclusividad apodíctico-deductiva (Popper-W. Stegmüller) interpretan esta última alternativa como demostrando que tales 
evidencias no pueden ser demostradas sin petitio principii. Apel sostiene, en cambio, que si superamos esta verdadera "abstractive fallacy” que supone omitir la dimensión pragmática; entonces, el hecho de que ciertas evidencias no pueden fundarse deductivamente (cuando además no pueden ser refutadas sin autocontradicción), no es ya prueba de la imposibilidad básica de una fundamentación última filosófica, sino precisamente conocimiento pragmáticotrascendental de los fundamentos no cuestionables de la argumentación con sentido.

Según esto, la afirmación cartesiana de la existencia de quien piensa, es desde el punto de vista lógico-formal, una tesis que se encuadra en el dilema de Stegmüller: no puede ni refutarse sin autocontradicción ni demostrarse sin petitio principii. Para Apel, precisamente por esto, la certeza del Cogito es una condición pragmático-trascendental de posibilidad del juego lingüístico de la argumentación. Dicho de otro modo, mediante el acto realizativo de dudar de mi exitencia refuto la propia proposición de duda. De manera general: aquellas sentencias fundamentales (Grund-Sätze) que no pueden ser negadas sin contradicción pragmática (o performativa), y cuya fundamentación sólo es posible en tanto son presupuestas, pueden considerarse como fundamentadas en forma definitiva.

Por la misma vía, es imposible afirmar la proposición: "Tú no existes"; quien así hablara, eliminaría con ello la posibilidad de hablar con un "tú", con lo cual fracasaría su acto de comunicación. Esto muestra que la certeza irrefutable del Cogito no se apoya, como supuso la filosofía de la conciencia, en un dato interior de la conciencia solitaria, sino sobre la comprensión de una "experiencia de situación a la vez reflexiva y comunicativa”, en la cual la inteligencia de sí mismo y de la existencia de los otros son "igualmente originarias", como ya lo habían visto G.H. Mead y M. Heidegger.

La argumentación filosófica revela pues, un juego transcendental del lenguaje, ya no ultrapasable, en el que se suponen: 
(1) ciertas reglas básicas de la lógica, (2) la existencia de un mundo real, (3) las reglas pragmático-trascendentales de la comunicación ideal, y por tanto también, (4) la existencia (actual o pasada) de una "comunidad real de comunicación" que proyecta la idea de una "Comunidad Ideal de Comunicación" que respalda a las reglas de comunicación ideal que constantemente tomamos como criterio. Dado que las aserciones y fundamentaciones últimas no tienen una validez limitada a una comunidad particular, sino que conllevan una pretensión de validez universal; Apel postula una Comunidad Ideal de Comunicación como condición de su posibilidad; esto es, concibe lo verdadero como el consenso en el seno de una tal comunidad.

Es claro que la comunidad ilimitada de comunicación no existe de hecho; ella representa una idea o postulado que actúa como orientación y criterio de corrección. Wolfang Röd (1989) lo explica ilustrativamente con una analogía, cuando dice:

Assim como Kant, por exemplo, explicou a validade universal dos axiomas geométricos com o auxilio da suposição de que o conceito euclidiano de espaço pertence não ao sujeito individual mas ao sujeito transcendental, também Apel procurou tornar concebivel a validade universal de tais sentenças, colocando como seu sujeito um princípio transcendental: a comunidade irrestrita de comunicação. Segundo Apel, aquele que julga com pretensão de validade universal pressupõe implicitamente uma comunidade ilimitada de interpretação como instância ideal de controle (Apel, 1973a, II, 215). Ela é, por assim dizer, a condição de possibilidade de juízos e argumentos universalmente válidos. Eis por que Apel também a qualifica como comunidade transcendental de comunicação. (RoD, 1989, p. 222)

Tenemos entonces que, si antes habíamos observado que Apel opera una "historización" de la filosofía trascendental, ahora observamos que realiza también una "socialización" del sujeto trascendental, aunque mediante el recurso a una "comunidad ideal", 
una socialización que tiene la virtud de no poder ser confundida con un relativismo.

A PRIMEIRA ANÁLISE DO PROBLEMA DE LA FUNDAMENTACION ÚLTIMA FILOSÓFICA EM K. O. APEL

Resumo: Em razão da complexidade e das grandes implicações do tema abordado por Apel em seu trabalho (ainda não traduzido para o português) El problema de la fundamentación última filosófica a la luz de una pragmática trascendental del lenguaje (1976) - uma verdadeira "camisa de força" desde seu próprio título -, este artigo, como sua primeira análise, se propõe a analisar as principais etapas e estratégias argumentativas usadas pelo autor, bem como indicar seus apoios teóricos mais importantes.

Palavras-chave: Trilema de Münchhausen, reflexão pragmático-transcendental, fundamentação filosófica não-metafísica.

\section{Notas}

1. Apel, K. O. El problema de la fundamentación última filosófica a la luz de una pragmática trascendental del lenguaje (Orig. Innsbruck, 1976). Traducido al español por Bernabé Navarro, Revista Dianoia, México, 198.

2. El “Trilema de Münchhausen” es, en realidad, un versión amplificada del dilema de Fries (Jacob Friedrich Fries, 1773-1843), que ya postulaba las dos primeras alternativas para la fundamentación última por vía deductiva. Fue a partir de esa versión original que ya Popper sostuvo que no podría haber fundamentación definitiva de conocimientos.

3. En su trabajo "De Kant a Peirce: la transformación semiótica de la Lógica trascendental” - original, Dordrecht (Holanda), 1970; e incluido después en K.O. Apel, Transformation der Philosophie, Frankfurt A.M. 1972, T. II) -, Apel había visto ya tal transformación en Ch. Peirce.

4. Wittgenstein (1987, p. 115): "Quien pretendiese dudar de todo, ni siquiera llegaría hasta la duda. El juego mismo del dudar presupone ya la certeza”. 
5. Idem, p. 114: "Quien no está cierto de ningún hecho, tampoco puede estar cierto del sentido de las palabras".

6. Putnam en Razón, verdad e historia hace una reinterpretación de la teoría kantiana de la verdad que la hace compatible con la concepción de Apel. Asimismo Putnam define verdad como "aceptabilidad racional idealizada", lo cual lo hace altamente convergente con la concepción que estamos viendo (PUTNAM, 1988, p. 59).

7. Independientemente de Apel, R. Haller en Über das sogenante Münchhausentrilemma in Ratio, 1974 (p. 126 y 133 y ss.), arriba a las mismas conclusiones.

8. Batley, W. Flucht ins Engagemene. München, 1964. p. 108 y ss. A conclusiones similares arriban, desde muy distintas perspectivas Steven Lukes (1977), Keith Dixon (1977), León Olivé (1981), e incluso P. Winch (1964) en la polémica inglesa sobre la pretendida universalidad de nuestra comprensión racional del mundo (reseñada por Habermas en Teoria de la Acción Comunicativa. Madrid: Tecnos, 1988, p. 82 a 99).

\section{Referências}

Albert, H. Tratado da razão crítica. Río de Janeiro: Tempo Brasileiro, 1976.

Apel, K. O. El problema de la fundamentación última filosófica a la luz de una pragmática trascendental del lenguaje. México: Revista Dianoia, n. XXI, 1976.

. La transformación de la filosofía. Madrid: Taurus, 1985.

Habermas, J. Teoría de la acción comunicativa. Madrid: Tecnos, 1988.

LENK, H. Philosophische Logikbegründig und rationaler Kritizismus. In: Lenk, H. Metalogik und Sprachanalyse. Freiburg, 1973.

Putnam, H. Razón, verdad e historia. Madrid: Tecnos, 1988. 
RoD, W. O problema de fundamentação última na filosofia contemporânea. Traduzido do original alemão por María Cecília M. de Carvalho. In: Paradigmas filosóficos da atualidade. Campinas: Papirus, 1989.

Wittgenstein, L. Sobre la certeza. Barcelona: Gedisa, 1987. 